\title{
A centralised telephone service for tobacco cessation: the California experience
}

Shu-Hong Zhu, Christopher M Anderson, Cynthia E Johnson, Gary Tedeschi, April Roeseler

\begin{abstract}
Objective-To provide an overview of the California Smokers' Helpline, an increasingly popular telephone program for tobacco cessation in California since 1992. As many states, regions, and nations are contemplating various telephone programs as part of large scale anti-tobacco campaigns, this paper presents a practical model.
\end{abstract}

Design-The Helpline provides Californians with free cessation services that include counselling, self help quit kits, and cessation related information. Services are provided in six spoken languages plus a line for the hearing impaired. The program is promoted statewide by media campaigns, health care providers, local tobacco control programs, and the public school system.

Setting-The Helpline is centrally operated through the University of California, San Diego and provides services statewide via telephone.

Results-The Helpline has served over 100000 tobacco users and has become the chief cessation resource for the Comprehensive Tobacco Control Program in California. Media was the most important referral source for Helpline callers $(50 \%)$, followed by health care providers $(20 \%)$. About one third of the callers were ethnic minorities and $17 \%$ were 24 years old or younger. Compared to California smokers in general, the callers were more dependent on nicotine and more likely to live with other smokers, but they were also more likely to have tried to quit recently and were more ready to try again. Two randomised trials have demonstrated the efficacy of the Helpline's counselling protocol.

California, San Diego, California, USA

$\mathrm{S}-\mathrm{H} \mathrm{Zhu}$

C M Anderson

C E Johnson

G Tedeschi

California Department of Health Services,

Tobacco Control

Section, Sacramento,

California, USA

A Roeseler

Correspondence to:

Dr Shu-Hong Zhu,
University of California, San

Diego, Mail Code 0905,

Diego, Mail Code 0905,
9500 Gilman Drive, La Jolla,

CA 92093-0905, USA;

eA 92093-0905, USA;
Conclusion-A centralised helpline operation can be an accessible and effective service for tobacco users and should be included in any large scale, comprehensive tobacco control program.

(Tobacco Control 2000;9(Suppl II):ii48-ii55)

Keywords: telephone helpline; tobacco cessation program

This paper describes the California Smokers' smoking cessation, that has been in operation for over seven years and has served more than 100000 California smokers and other tobacco users. As many decision makers are weighing Helpline, a statewide telephone service for the value of tobacco helplines and others are in the process of implementing one, this paper aims to provide information that will be useful for policy makers as well as for practitioners and researchers. It presents the rationale for a tobacco helpline and describes the basic structure of the California Smokers' Helpline, its role in the Comprehensive Tobacco Control Program in California (CTCPC), the population it serves, and the evidence of its efficacy. The potential of such a helpline for future tobacco control practice and research is also discussed.

Why a telephone counselling program? There are many programs for tobacco cessation. Why do we need a telephone program? From a public health service perspective, there are at least four good reasons.

First, smokers are more likely to use a telephone service than a face-to-face program, because the former is more convenient for them. When offered the choice between the two forms of service, most smokers (75-85\%) prefer telephone programs. ${ }^{12}$ Because the low use of cessation services has hampered the usefulness of most programs, ${ }^{3}$ a more accessible form of service such as telephone counselling is much preferred when one is considering establishing a new program.

Second, a telephone operation allows services to be centralised in one site, which permits a certain economy of scale. In the case of the California Smokers' Helpline, a centralised operation for the whole state of California makes it economically feasible for the Helpline to operate every weekday from 9 am to $9 \mathrm{pm}$ and Saturday from 9 am to $1 \mathrm{pm}$. Most traditional cessation programs are offered infrequently and require participants to wait for the next upcoming group meeting. ${ }^{4}$ The centralised operation of the California Smokers' Helpline makes it feasible to staff the Helpline year round with well trained counsellors who provide counselling in six different languages, so that smokers and other tobacco users across the state can have access to quality service in their preferred language. Standardised training, quality control, and evaluation systems are better managed in a single setting, as well. It would have been cost prohibitive to staff individual local cessation programs across California in a similar manner.

Third, a centralised operation makes it easier to promote the Helpline services. This is particularly true when coordinated anti- 
tobacco efforts exist on a statewide or nationwide level. The California Smokers' Helpline, for example, is the favourite cessation program promoted by the statewide media campaign partly because it is easy to tag the same toll-free number (in each language) on numerous media spots statewide. If the media campaign were to tag the numbers of individual local programs, it would have to do so for many different numbers, representing a variety of local cessation programs, to the media spots according to the locality in which they are aired. This would have been impractical. Thus, many large scale anti-tobacco media campaigns in recent years have promoted centrally operated helplines. ${ }^{5-9}$

Last, the telephone can be effective in proactively recruiting tobacco users into a cessation program. ${ }^{10}$ This is particularly useful with certain high risk populations, such as women who continue to smoke during pregnancy and do not attend any cessation program to quit smoking. Studies have shown that the majority of these pregnant smokers accepted telephone counselling when they were proactively contacted by nurses who offered the counselling by phone. $^{11}$ As the cost effectiveness of providing cessation service to groups such as pregnant smokers is significant, ${ }^{12}{ }^{13}$ a program's ability to proactively recruit smokers and counsel them by phone makes it a much more attractive option from a public health perspective.

Recognising the potential of a centralised telephone service centre, several states in the US have established statewide tobacco helplines-California, Massachusetts, Arizona, Michigan, and Oregon. Some regions or nations have regional or national helplines. ${ }^{6} 714$ Many others, especially those states in the US which have been allocated funds from the recent national tobacco settlement or from cigarette taxes, are contemplating similar programs. However, few reports describe how a helpline operates and how it can be used in conjunction with other tobacco control activities. This paper is intended to fill that gap by presenting one model-the California Smokers' Helpline.

\section{The California Smokers' Helpline}

The California Smokers' Helpline is a statewide program funded through the California Tobacco Tax Initiative (Proposition 99), via the California Department of Health Services, Tobacco Control Section. The Helpline is administered by the Cancer Center of the University of California, San Diego (UCSD). Program services, offered free of charge to all Californians, include individual counselling, self help materials, information related to tobacco cessation, and referral to local services. To maximise accessibility, separate toll free numbers are provided in English, Spanish, Vietnamese, Korean, and Chinese (for Mandarin and Cantonese speakers). Also provided is a line for the hearing impaired and another line for those who use chewing tobacco or snuff. The Helpline has been in operation since 1992 and has served over 100000 smokers and other tobacco users. To support an operation of this magnitude and to ensure the quality of service, the program is structured with three interrelated elements: intake, counselling, and evaluation.

INTAKE

When a call comes in to the Helpline, a staff member conducts a brief intake interview, gathering information about tobacco use, previous quit attempts, attitudes about quitting, and demographics. The caller is presented with a menu of service options. Some callers do not use tobacco themselves but are simply requesting information for family or friends; information is sent to them promptly. Most callers are smokers or other tobacco users who are contemplating quitting, in which case the intake staff assesses their readiness to quit. Those who are ready to quit within one week can choose either self help materials or individual counselling. Those who are not ready receive motivational materials designed to encourage them to take the next step. Regardless of which service is selected, the Helpline mails a list of cessation programs available in the caller's county of residence so that he or she can choose from those programs as well.

\section{COUNSELLING}

Callers who choose counselling may receive up to seven sessions of counselling over a period of two months. Once the caller is assigned to a counsellor, the counsellor initiates each session. The same counsellor follows up with the caller through the whole process. A thorough discussion of the counselling protocol, including the training of the counsellors, can be found in the article by Zhu and colleagues. ${ }^{15}$

\section{EVALUATION}

A sample of callers is chosen for evaluation after receiving services. The evaluation determines which clients have tried to quit, whether they are currently using tobacco, and how satisfied they are with the services received. The evaluation provides feedback to the Helpline about its efficacy and conveys timely information about public perception of the service. From the start of the project, the Helpline has emphasised the importance of gathering evidence of the program's efficacy. Although the counselling protocol was based on an earlier clinical trial, ${ }^{16}$ it was believed that its efficacy should be replicated in a service setting for several reasons. First, telephone counselling is a new mode of intervention, and only a few studies have demonstrated its efficacy. ${ }^{17}$ When a protocol is translated from a controlled research design to a large scale service, replication is necessary to ensure that program efficacy has not dissipated. ${ }^{18} 19$ Second, the amount of money that the Helpline spends has been increasing over the years because of the growing number of callers requesting service (an average of $\$ 1.3$ million annually in the last seven years). Because interventions for tobacco cessation are often criticised for their lack of 
efficacy, up to date evidence of efficacy for the program is important considering the uncertain climate of funding for tobacco control programs. ${ }^{20}$ Third, there have been many developments in tobacco control strategies and activities in California. Evaluation of the Helpline as the only statewide cessation program provides timely data about smokers' responses to California's ongoing campaign for tobacco cessation, which is useful to the funding agency in planning future cessation efforts in California.

\section{Smokers' Helpline in CTCPC}

One goal of CTCPC is to increase the quitting among current tobacco users. ${ }^{21}{ }^{22}$ This includes the effort to motivate them to quit by changing the social norms to render smoking behaviour socially less desirable and to provide assistance for those who want to quit. The California Smokers' Helpline plays an important role in assistance to quitting. Because the Helpline is accessible to all Californians who have telephones, it is an ideal program to be promoted by multiple groups and through multiple channels. The following describes four major groups that the Helpline has worked with over the years in a statewide effort to encourage and assist tobacco users to quit. They are the anti-tobacco mass media campaign, the health care providers, the local tobacco control programs, and the school system.

\section{HELPLINE AND THE MASS MEDIA CAMPAIGN}

The major goal of the anti-tobacco media campaign in California is to denormalise tobacco use in society. ${ }^{52}$ By influencing the public's perception about tobacco use, the campaign also motivates current users to quit. The organisers of the media campaign considered it important to send a message, along with the anti-tobacco agenda, that assistance is available for those who want to quit. To that end, the campaign chose the Helpline as the cessation service to be promoted statewide.

The media promotion of the Helpline involves making specific media spots, in different languages, to encourage tobacco users to call the Helpline. These media spots were the main channels of information about the existence of a Helpline at the beginning of the program. For example, from August 1992 to December 1993, 72\% of the 16720 callers reported that they heard about the Helpline from the media campaign.

The effect of the media campaign for the Helpline, of course, is not just on those who actually called, though it is hard to measure for those who did not call. Ossip-Klein and her colleagues have shown, in a randomised study, that a majority of tobacco users who were informed of the existence of a helpline did not call for counselling services. However, the group that knew of the existence of a helpline was more likely to make a quit attempt than the group that did not know about it. ${ }^{23}$ This suggests that it is beneficial to tag helpline numbers to media spots whenever it is appropriate. This is exactly what the California media campaign has done and, as mentioned earlier, the Helpline's centralised operation makes it easy for the statewide media campaign to tag its numbers.

The media campaign and the Helpline message can sometimes work together to produce a synergistic effect. An example of this is the effort to reach Asian American and Pacific Islanders (AAPIs) whose native language is not English. These recent immigrants are generally not inclined to seek public health assistance, partly because of their low acculturation and lack of language proficiency. The Helpline's initial effort to contract with AAPI community programs to promote the Helpline services among these groups was not successful. From 1994 to 1997 , only seven calls per month came through the Asian language 800 lines (Vietnamese, Korean, Mandarin, and Cantonese). However, when media spots combining a secondhand smoke message with the message of help were aired in these Asian languages in 1998, they generated 234 calls each month (for a 12 month period) to these Asian language lines. More importantly, about $40 \%$ of the calls that came through the Asian language lines were proxy calls, compared to only $6 \%$ of calls to the English language line. ${ }^{24}$ (Proxy callers are those do not use tobacco themselves, but who call on behalf of family or friends who smoke). This suggests that, in these communities, the secondhand smoke campaign had mobilised non-smokers as well as smokers to take actions that were intended by the media campaign's planners. The fact that the media spots were tagged with a helpline number made it easier for these non-smokers to get involved. At the same time, this media campaign successfully brought the helpline message across to these communities that had been, so far, difficult to reach.

While media has been the chief promoter of the California Smokers' Helpline, it was important that the Helpline did not get completely dependent on a media campaign, which is an expensive operation. Over the years, the Helpline has striven and succeeded in increasing its reach to smokers through other channels. As shown in fig 1, the monthly calls to the Helpline due to non-media sources were about 75 in 1992 when the project started. This was increased to about 360 in the years 1993 to 1996. Then, in 1997, the

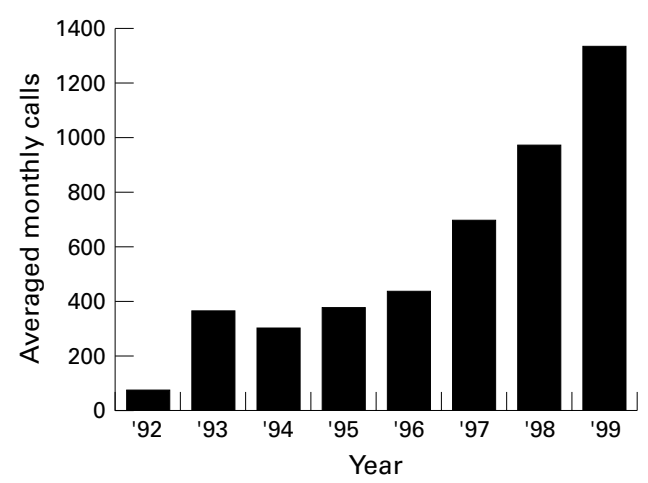

Figure 1 Monthly calls to the Helpline as a result of non-media sources 
monthly calls due to non-media sources increased to about 700 and continued to rise to a little over 1300 in 1999 . The following gives a brief description of how the Helpline worked with three major non-media groups-health care providers, local tobacco control programs, and the school system-to promote its services.

HELPLINE AND HEALTH CARE PROVIDERS

Approximately $70 \%$ of smokers visit health care providers at least once a year. This presents a good opportunity to help them quit. The Agency for Health Care Policy and Research (now the Agency for Healthcare Research and Quality) recommends, in its clinical practice guidelines, that physicians ask about their patients' smoking status at each visit, advise those who are smoking to quit, assist them by prescribing nicotine replacement treatment (NRT) when appropriate, and provide personal counselling. ${ }^{25}$ However, some difficulties in implementing the guidelines are that physicians are not used to providing behavioural counselling and, even if they want to, their time is limited. ${ }^{26}{ }^{27}$ Furthermore, there are difficulties on the system level because physicians are not reimbursed for time spent counselling their patients about tobacco cessation. ${ }^{27}$ Thus, though it is an attractive idea to work with physicians, there are many practical obstacles in actual implementation.

Having no direct access to the health care system on an organisational level, the California Smokers' Helpline started with individual physicians. Working through conferences, special events (for example, the American Cancer Society's Great American Smokeout), and other tobacco control programs that work directly with health care providers, the Helpline presents to physicians the idea that the telephone counsellors can become an extension of their service in the area of tobacco cessation. In this scenario, the physicians "ask" and "advise" and the Helpline provides counselling. All the physicians need to do is give their patients the Helpline's 800 numbers after advising them to quit. (To make it as easy as possible for the physicians, the Helpline provides wallet cards with the toll free numbers printed on them, which can be given to patients during office visits.) Once the patient enrolls in telephone counselling, the counsellor proactively follows up with the patients to reduce the probability that they will drop out of the process. ${ }^{28}$

The Helpline offers positive reinforcement to health care providers who ask, advise, and refer by periodically sending thank you letters to convey the message that their referral has made a difference. The Helpline also uses every opportunity to motivate physicians. For example, when Medi-Cal decided that its recipients statewide could receive NRT for free if they enrolled in the Helpline, the Helpline worked hard to help physicians who prescribed NRT. Recognising that physicians may have time to prescribe medication but not to provide behavioural counselling, the Helpline uses the opportunity to demonstrate to physicians that
Helpline counsellors will extend the physician's service in order to ensure greater compliance. The data from this collaborative effort indicate that those NRT users who received follow up counselling from the Helpline were more likely to stay abstinent than those who dropped out of counselling after receiving their free NRT. ${ }^{29}$ Currently, more than 2000 health care providers are referring their patients to the Helpline. Altogether, they have referred over 20000 patients since the Helpline's inception.

Based on the success the Helpline had with individual physicians, the Helpline presented the data to large managed care organisations (MCOs). Given that the service is free of charge for all Californians, it was expected that the Helpline would be a very attractive option for insurance groups. In reality, it took a long time before any major MCOs expressed interest in participating, although a few smaller insurance groups started routinely referring patients to the Helpline in 1993. After almost seven years of Helpline operation, one large MCO, Kaiser Permanente of Northern California, decided that its patients could have cessation medication (NRT, bupropion) if they enroll in the California Smokers' Helpline. Now that a group called Next Generation (funded by the Robert Wood Johnson Foundation) is actively working with an extensive network to promote the collaboration between MCOs and the tobacco control programs in California, ${ }^{30}$ it is expected that more MCOs will follow suit.

HELPLINE AND THE LOCAL TOBACCO CONTROL PROGRAMS

Another group with which the Helpline worked closely is comprised of those tobacco control programs that receive funding from the California Department of Health Services, Tobacco Control Section (Proposition 99 funds). They include the county health departments and a variety of groups receiving tobacco control funding on a competitive basis. Some of these grantees run local projects (for example, working with health care providers at community clinics to help pregnant smokers), while others operate on a statewide basis (for example, the multi-ethnic networks for tobacco control). Many of them work with current smokers, but they often lack the capability of providing counselling services. The Helpline has become an ideal program to which to refer because it is multilingual and equally accessible everywhere in California. For example, many of the county health departments were involved in the education work on $\mathrm{AB} 13$, a bill that banned smoking in the workplace in California in early 1994. When these agencies presented the new rules to companies' employees, they informed them about the Helpline at the same time. It is the same principle that was applied in the media campaign discussed earlier: while presenting information that motivates tobacco users to quit (in this case, the new ban), the agencies also send the message that help is available for those who want to quit. 
To keep this group of tobacco control workers motivated to promote Helpline services, the Helpline sends demographic data to grantees interested in knowing the statistics on callers from their own county within any particular time period. With time, this network of tobacco control grantees grew strong and eventually the Department of Health Services required that all tobacco control grantees include the Helpline's 800 numbers in their printed materials. Thus, the Helpline has become part of the infrastructure for the overall tobacco control program conducted by the Department of Health Services. ${ }^{22}$ In fact, this referral network has grown to include health agencies that may not receive any grants from the Health Department, such as the American Cancer Society, American Lung Association, and American Heart Association.

HELPLINE AND THE SCHOOL SYSTEM

In recent years, the Helpline has worked to expand its services to the California public schools that receive tobacco control money administered by the California Department of Education. The Department of Education funds numerous tobacco use prevention education (TUPE) projects in school districts across California. Although these projects are not part of the campaign conducted by the Department of Health Services, they are an important element of the overall tobacco control program envisioned by those who crafted the tobacco tax initiative (Proposition 99). Most of the TUPE projects contain activities in their scope of work to help current smokers quit. However, the local counselling services for adolescents often lack the resources required to meet this objective. The Helpline did not provide counselling for teens until 1996, and therefore had not promoted its services to schools before then. When the statewide Helpline for adolescents finally hap-

Table 1 How did the Helpline callers hear about the program (August 1992 to December 1999)

\begin{tabular}{|c|c|c|c|c|}
\hline & $\begin{array}{l}\text { Mass media } \\
(n=54852)\end{array}$ & $\begin{array}{l}\text { Health care } \\
\text { providers } \\
(n=21760)\end{array}$ & $\begin{array}{l}\text { Family/friend } \\
(n=11902)\end{array}$ & $\begin{array}{l}\text { Other } \\
(n=21728)\end{array}$ \\
\hline Mean & $49.8(0.15)$ & $19.7(0.12)$ & $10.8(0.09)$ & $19.7(0.12)$ \\
\hline \multicolumn{5}{|l|}{ Sex } \\
\hline Male & $60.1(0.22)$ & $14.2(0.15)$ & $9.6(0.13)$ & $16.1(0.16)$ \\
\hline Female & $40.6(0.20)$ & $24.6(0.18)$ & $11.9(0.13)$ & $23.0(0.17)$ \\
\hline \multicolumn{5}{|l|}{ Age (years) } \\
\hline$<18$ & $36.7(0.71)$ & $6.3(0.36)$ & $13.2(0.50)$ & $43.7(0.73)$ \\
\hline $18-24$ & $63.3(0.41)$ & $9.2(0.24)$ & $9.7(0.25)$ & $17.9(0.33)$ \\
\hline $25-44$ & $52.6(0.20)$ & $18.8(0.16)$ & $10.6(0.12)$ & $18.1(0.16)$ \\
\hline $45-64$ & $39.1(0.30)$ & $29.0(0.28)$ & $11.4(0.20)$ & $20.6(0.25)$ \\
\hline $65+$ & $37.3(0.78)$ & $30.8(0.74)$ & $13.0(0.54)$ & $18.9(0.63)$ \\
\hline \multicolumn{5}{|l|}{ Ethnicity } \\
\hline White & $41.5(0.19)$ & $24.5(0.17)$ & $12.1(0.13)$ & $21.8(0.16)$ \\
\hline African-American & $56.1(0.48)$ & $16.4(0.36)$ & $8.9(0.28)$ & $18.6(0.37)$ \\
\hline \multicolumn{5}{|l|}{ Hispanic } \\
\hline English line & $50.7(0.59)$ & $14.5(0.41)$ & $10.4(0.36)$ & $24.4(0.50)$ \\
\hline Spanish line & $81.2(0.41)$ & $3.8(0.20)$ & $7.6(0.28)$ & $7.4(0.27)$ \\
\hline \multicolumn{5}{|l|}{ AAPI } \\
\hline English line & $55.1(0.94)$ & $10.3(0.57)$ & $10.5(0.58)$ & $24.2(0.81)$ \\
\hline Asian lines & $93.7(0.34)$ & $1.2(0.15)$ & $3.0(0.24)$ & $2.2(0.20)$ \\
\hline Others & $39.4(0.64)$ & $24.8(0.56)$ & $12.7(0.43)$ & $23.1(0.55)$ \\
\hline \multicolumn{5}{|l|}{ Education } \\
\hline$\leqslant 12$ years & $43.6(0.23)$ & $25.2(0.20)$ & $12.7(0.15)$ & $18.5(0.18)$ \\
\hline$>12$ years & $53.2(0.22)$ & $17.1(0.16)$ & $9.5(0.13)$ & $20.2(0.18)$ \\
\hline \multicolumn{5}{|l|}{ County of residence } \\
\hline Urban & $63.4(0.18)$ & $11.0(0.12)$ & $8.8(0.10)$ & $16.9(0.14)$ \\
\hline Rural/mixed & $23.4(0.22)$ & $36.7(0.25)$ & $14.7(0.18)$ & $25.2(0.22)$ \\
\hline
\end{tabular}

Only first time callers are included in this analysis; data presented as \% (SE). pened, it immediately became a popular referral resource among TUPE coordinators. Now the Helpline regularly provides up-to-date information about the Helpline to all the TUPE coordinators and many of the health educators across the state (approximately 800).

To show how these joint promotional efforts have worked, table 1 presents data on how the 110242 first time callers from August 1992 to December 1999 were referred. Overall, about $50 \%$ of callers reported that they had heard about the program from media sources and about $20 \%$ said that their health care providers referred them. About $11 \%$ reported that they had heard about the Helpline from family or friends, who may have used the Helpline themselves or simply seen the media spots and passed the information along. Because each of the rest of the individual sources of information was reported by fewer than $10 \%$ of callers, they are grouped into "other," which accounts for about $20 \%$ of the calls.

Table 1 shows some interesting differences across several demographic dimensions. Male callers are more likely than female callers to have heard about the Helpline through the media, while females are more likely than males to have heard about it from their health care providers. The latter may be related to the fact that females are more likely to visit their physicians. ${ }^{31}$ With callers under 18 years as an exception, referral by media decreases with age, while referral by health care providers increases. Of those under 18 , only about $37 \%$ were referred by the media. This is related to the fact that the media did not promote the Helpline at all among this age group during the first four years of operation because counselling services for this group did not exist until 1996. For this underage group, about $12 \%$ reported that their school referred them. Another 19\% reported that they got the Helpline's number from a gold card, which is made to resemble a credit card bearing the Helpline numbers and is distributed primarily at schools. Both of these figures are included in the "other" category in table 1 .

Different ethnic and linguistic groups rely on the media for information about the Helpline to different extents. White callers were less dependent on the media than non-white callers. Only $41.5 \%$ of white callers were referred by the media, compared to $56.1 \%$ of African-American callers. Even higher percentages of Hispanic and AAPI callers were referred by the media (taking both English and non-English speaking callers in those groups together). With respect to language, English speaking callers were less dependent on the media than non-English speaking callers. Over $80 \%$ of Spanish language callers were referred by media and less than $4 \%$ by health care providers. Of Asian language callers, almost 94\% were referred by media and only $1.2 \%$ by providers.

Callers with less education are more likely to have heard about the Helpline from health care providers. This may reflect the fact that the Helpline receives a significant number of callers who are Medi-Cal patients $(43.8 \%$ of 
Helpline callers since 1998). Medi-Cal patients tend to have a lower education level. Many of them call the Helpline as a result of their health care provider's referral because they learn from them that they can get free NRT if they enroll in the Helpline. In this way, the Helpline reaches a segment of the population with a lower socioeconomic status who are in greater need of assistance, because they typically are heavier smokers and do not seek help as often as those with a higher socioeconomic status. ${ }^{32}$

Finally, callers from urban counties are significantly more likely to hear about the Helpline from the media than those in rural and mixed counties. The main sources of referral for the latter are health care providers. As will be seen later, this difference is not caused by a lower participation by residents of non-urban areas. In fact, those in rural and mixed counties are more likely to call than those in urban counties, and are more likely to have heard about the Helpline from their providers. This suggests that rural health care providers more actively refer patients to the Helpline than providers in urban areas.

\section{Who used the Helpline?}

Overall, the Helpline received over 117000 calls from August 1992 to December 1999. About $7 \%$ were proxy calls (calls on behalf of friends or family), and another $4 \%$ were repeat calls (those who called more than once in a year). Table 2 presents the demographics of callers, excluding proxy and repeat callers. The demographics of smokers from the 1996 California Tobacco Survey (CTS) are also presented for comparison. The data from CTS are weighted according to census population.

Compared to the general smoking population in California, Helpline callers are more likely to be female. This is consistent with sex differences observed in other studies on participation in cessation programs. ${ }^{32}$ Overall, tobacco users of all ages are well represented in

Table 2 Helpline callers compared to general smoking population in California: demographics

\begin{tabular}{|c|c|c|}
\hline & $\begin{array}{l}\text { California } \\
\text { Smokers'Helpline } \\
(n=103070)\end{array}$ & $\begin{array}{l}\text { State of California } \\
(' 96 \text { CTS }) \\
(n=14229)\end{array}$ \\
\hline \multicolumn{3}{|l|}{ Sex } \\
\hline Male & $48.0(0.16)$ & $57.1(0.41)$ \\
\hline Female & $52.0(0.16)$ & $42.9(0.41)$ \\
\hline \multicolumn{3}{|l|}{ Age (years) } \\
\hline$<18$ & $3.9(0.06)$ & $2.5(0.14)$ \\
\hline $18-24$ & $12.8(0.10)$ & $13.6(0.34)$ \\
\hline $25-44$ & $56.3(0.15)$ & $50.5(0.46)$ \\
\hline $45-64$ & $23.7(0.13)$ & $25.5(0.41)$ \\
\hline $65+$ & $3.4(0.06)$ & $7.8(0.27)$ \\
\hline \multicolumn{3}{|l|}{ Ethnicity } \\
\hline White & $63.3(0.15)$ & $58.7(0.55)$ \\
\hline African-American & $10.4(0.10)$ & $8.3(0.27)$ \\
\hline Hispanic & $14.9(0.11)$ & $22.1(0.45)$ \\
\hline AAPI & $5.9(0.07)$ & $7.4(0.28)$ \\
\hline Others & $5.5(0.07)$ & $3.5(0.23)$ \\
\hline \multicolumn{3}{|l|}{ Education } \\
\hline$\leqslant 12$ years & $48.4(0.16)$ & $58.6(0.47)$ \\
\hline$>12$ years & $51.6(0.16)$ & $41.4(0.47)$ \\
\hline \multicolumn{3}{|l|}{ County of residence } \\
\hline Urban & $64.9(0.15)$ & $75.4(0.42)$ \\
\hline Rural/mixed & $35.1(0.15)$ & $24.6(0.42)$ \\
\hline
\end{tabular}

The data (presented as \% (SE)) cover the period from August 1992 to December 1999. All proxy and repeat callers are tobacco screener survey, weighted by population. the Helpline; in fact, Helpline callers are slightly younger than the general smoking population. This is significant because help seeking for quitting tends to be less prevalent among those who are younger. ${ }^{33}$

With regard to ethnicity, African-American callers are overrepresented while the reverse is true of Hispanic callers. The underrepresentation of Hispanic callers is mostly accounted for by low media promotion in the Spanish language during the period of 1997 to 1999. As shown in an earlier study, ${ }^{33}$ when the media had active Spanish language promotion, Hispanic callers were well represented. As was indicated in table 1, Spanish speakers relied heavily on the media as a source of information about the Helpline. As the majority of Hispanic callers used the Spanish line, these data suggest that sufficient media coverage in Spanish is necessary to increase utilisation of the Helpline by the Hispanic community. Those of AAPI background are also somewhat underrepresented, although in 1998 there was a significant increase in participation by AAPI's owing to a successful media campaign targeting those communities. $^{24}$ Overall, minority callers are underrepresented (by about 5\%). However, with a greater concentration of ethnically and linguistically targeted advertising, it seems clear that the percentage of ethnic minority smokers could be raised.

Helpline callers are somewhat more educated than the general smoking population in California. This is true with other cessation programs. $^{32}$ Although the Helpline has successfully campaigned among the Medi-Cal population, the data indicate that more needs to be done to reach less educated tobacco users.

Interestingly, those from rural and mixed counties are more likely to call than those from urban counties. In fact, more than a third of callers are from rural and mixed counties, while only about a quarter of the state's smokers reside there. An earlier study observed the same phenomenon, ${ }^{33}$ although it is more pronounced in the current data. As shown in table 1, the high level of participation by rural smokers is correlated with the fact that rural health care providers have been actively referring.

Table 3 shows other characteristics of Helpline callers. Compared to smokers in general, Helpline callers are more addicted. A much higher percentage of Helpline callers smoke 15 cigarettes or more per day. They are also more likely to smoke their first cigarette within 30 minutes of waking. Both of these are good measures of nicotine dependence. ${ }^{34}$ Compared to smokers in general, those calling the Helpline are also more likely to have another smoker in the household, which is a predictor of relapse in quitting. ${ }^{32}$ In other words, Helpline callers tend to be in a less advantageous position both in terms of level of nicotine addiction and of environmental cues for relapsing after quitting.

On the other hand, Helpline callers are more experienced in their quitting process than smokers in general. Almost three quarters of 
Table 3 Helpline callers compared to general smoking population in California: smoking variables

\begin{tabular}{lll}
\hline & $\begin{array}{l}\text { California Smokers' } \\
\text { Helpline }(n=100048)\end{array}$ & $\begin{array}{l}\text { State of California } \\
\text { ('96 CTS })(n=8710)\end{array}$ \\
\hline Smoke $\geqslant 15$ cigarettes/day & $70.1(70.0$ to 70.2$)$ & $44.6(43.0$ to 46.1$)$ \\
Smoke within 30 minutes* & $70.1(69.8$ to 70.4$)$ & $55.8(54.5$ to 57.1$)$ \\
Have other smokers in the house & $46.1(45.8$ to 46.4$)$ & $39.0(37.4$ to 40.6$)$ \\
Tried to quit in the last year & $72.6(72.2$ to 73.0$)$ & $51.4(50.2$ to 52.6$)$ \\
Ready to quit in a month & $93.2(93.0$ to 93.4$)$ & $13.8(12.6$ to 15.0$)$
\end{tabular}

The data (presented as \% (95\% confidence intervals)) cover the period from August 1992 to December 1999. All proxy and repeated callers are excluded. The 1996 CTS data are from the 1996 California tobacco screener and extended surveys, weighted by population.

${ }^{\star}$ Only daily smokers were asked this question.

callers $(72.6 \%)$ have tried to quit within the last 12 months, compared to $51.4 \%$ of the general smoking population. Most Helpline callers, $93.2 \%$, are ready to quit within a month, compared to only $13.8 \%$ of the general smoking population. These measures are associated with higher quit rates. ${ }^{35}$ Thus, Helpline callers are more likely to quit when compared to smokers in general, as measured by motivational benchmarks.

\section{Is the Helpline service helpful?}

With the high level of readiness to quit using tobacco among Helpline callers (93.2\%), a natural question is whether the Helpline service is helping them at all, or would these highly motivated callers quit without help anyway?

The data indicate that when Helpline callers make a quit attempt using only the Helpline's self help materials, their 12 month abstinence rate reaches $14.7 \%{ }^{16}$ This success rate was replicated in a second study. ${ }^{36}$ It is similar to the rate identified by a meta-analysis for most self care programs. ${ }^{37}$ However, this is much higher than the $7.0 \%$ abstinence rate observed in the California smoking population who attempted to quit without any help. ${ }^{38}$ As impressive as this difference is, it is difficult to attribute it all to the self help materials. It is more likely that Helpline callers are more motivated and that accounts for most of the difference. This is the problem inherent in evaluating the effect of self help materials - the lack of a control group that receives nothing. Thus, although those using the Helpline materials do well when compared to those who seek no help, the exact magnitude of the effect of the materials is not clear. Most likely, the net effect of the self help materials is small, ${ }^{39}$ which, however, is not to say that the materials are unnecessary. On the contrary, self help materials need to be an integral part of any population based approach to tobacco cessation. ${ }^{39}$

The effect of the telephone counselling is easier to evaluate. An earlier randomised trial demonstrated that providing Helpline counselling doubled the success rate, relative to that which was achieved by callers who received only self help materials. ${ }^{16}$ This result has been replicated in a second randomised trial, which was embedded in the current service setting of the Helpline. ${ }^{36}$ Both trials were conducted with smokers who said they were ready to quit and who were randomly assigned to one or the other condition (each condition had more than
1000 subjects). Thus, there is strong evidence that the Helpline counselling is effective for those who are ready to quit. So the Helpline currently focuses its counselling efforts on those smokers. Studies testing protocols for those who are not ready to quit are underway.

\section{Helpline as a laboratory for public health research}

Last, the Helpline is an excellent setting in which to conduct public health research. In such a program, it is possible to recruit sufficient numbers of subjects to find answers to challenging research questions. For example, studies on the effectiveness of behavioural interventions for adolescent smokers are often hampered by the difficulty of recruiting subjects. The Helpline, however, has been able to recruit over 1000 adolescent smokers into an ongoing study. Each of the two randomised trials with adult smokers mentioned earlier had more than 3000 subjects. The positive results obtained from such trials provide confidence that the interventions tested are indeed helpful. Currently, another large trial with pregnant smokers is underway.

The Helpline also provides opportunities to test interventions in a setting closer to the "real world". For example, many insurance plans require that tobacco users receive behavioural interventions if they want their NRT covered, on the assumption that NRT would not have much effect in the real world if it were used without behavioural counselling. This line of thinking is based in part on the fact that most clinical trials of NRT to date have included an element of behavioural intervention, or at least frequent contact with clinical staff. ${ }^{25}$ However, the extent to which behavioural interventions increase the success rate for those who use NRT in a real world setting has not actually been tested. With thousands of smokers and other tobacco users calling the Helpline each month and more than $35 \%$ of them using NRT, it would be feasible to test in a rigorous design the value of counselling as an adjuvant to NRT. The California Smokers' Helpline continues to investigate questions such as these, both to improve its own service and to add to the field of knowledge about effective cessation treatments.

Many individuals have made substantial contributions to the California Smokers' Helpline. The authors especially wish to thank Michael Byrd, Sharon Cummins, Denise Freeman, Elsa Gutiérrez-Terrell, Susan Hian, Eva Martinez, Judith Mills, Laura Muesse, Mary Lou Munguía, and Judy Quan, who were instrumental in the development of the project. The authors instrumental in the development of the project. The authors also wish to thank Jichao Sun for providing statistical assistance. Funding was provided by the California Department of Health Services, Tobacco Control Section, under grants 90-10961,
92-15416, and 96-27049.

1 McAfee T, Sofian N, Wilson J, Hindmarsh M. The role of tobacco intervention in population-based health care. $\mathrm{Am}$ f Prev Med 1998;14:46-52.

2 Wassum K. Free and clear program. Paper presented at the Third Annual Conference on Addressing Tobacco Control in Managed Care: Shaping Solutions for Today's Health Care Market Atlanta, Georgia, 2000.

3 Chapman S. Stop-smoking clinics: a case for their abandonment. Lancet 1985; i: 918-20.

4 Schwartz JL. Review and evaluation of smoking cessation control methods: the United States and Canada, 19781985. US Department of Health and Human Services, National Institute of Health. NIH Publication No. 87-2940, 1987. 
5 Stevens C. Designing an effective counteradvertising campaign-California. Cancer 1998;83:2736-41.

. First report of the evaluation of the national quitline service. Report to the Australian Ministerial Tobacco Advisory Group, National Tobacco Advisory Group, National Tobacco Campaign. Adelaide, South

7 Australia, South Australian Health Commission, 1997. the 10th World Conference on Tobacco and Health. Beijing: China, 1997

8 Platt S, Tannahill A, Watson J, Fraser E. Effectiveness of antismoking telephone helpline: follow up survey. $B M \mathcal{F}$ 1997;314:1371-5.

9 Peters L. An evaluation of the Quit 4 Life smoking cessation program. A report to Health Canada. Ottawa, Ontario: Publisher 1995 .

10 McDonald PW. Population-based recruitment for quitsmoking programs: an analytic review of communication variables. Prev Med 1999;28:545-57.

11 Ershoff D, Quinn VP, Boyd NR, et al. The Kaiser Permanente prenatal smoking-cessation trial. Am $\mathcal{f}$ Prev Permanente prenatal
Med 1999;17:161-8.

12 Lightwood JM, Phibbs CS, Glantz SA. Short-term health and economic benefits of smoking cessation: low birth weight. Pediatrics 1999;104:1312-20.

13 Windsor RA, Lowe JB, Perkinds LL, et al. Health education for pregnant smokers: its behavioural impact and cost benefit. Am f Public Health 1993;83:201-6.

14 Zeeman G. Seven years of smoking cessation campaigns in the Netherlands. Paper presented at the 10th World Conference on Tobacco and Health. Beijing: China, 1997.

15 Zhu S-H, Tedeschi G, Anderson C, Pierce J.P. Telephone counseling for smoking cessation: what's in a call? f Counsel Devel 1996;75:93-102.

16 Zhu S-H, Stretch V, Balabanis M, et al. Telephone counseling for smoking cessation: effects of single-session and multiple-session interventions. F Consult Clin Psychol 1996;64:202-11.

17 Lichtenstein E, Glasglow RE, Lando HA, et al. Telephone counseling for smoking cessation: rationales and metacounseling for smoking cessation: rationales and metaTheory and Practice 1996;11:243-57.

18 Gilbert JP, Mosteller F, Turkey J. Steady social progress requires quantitative evaluation to be searching. In: Abt CC, ed. The evaluation of social programs. Beverly Hills Sage, 1976.

19 Boruch RF. Randomized experiments for planning and evaluation: a practical guide. Thousand Oaks:Sage, 1997.

20 Husten C. Smoking cessation on the national and state levels. Paper presented at the Sixth Annual National Conference on Tobacco Control. Orlando, Florida, 1999.

21 Bal DG, Kizer KW, Felton PG, et al. Reducing tobacco consumption in California. Development of a statewide anti-tobacco use campaign. $\mathcal{F} A M A$ 1990;264:1570-4.

$22 \mathrm{CDHHS} / \mathrm{TCS}$. A model for change: the California Experience in tobacco control. California Department of Health Services, Tobacco Control Section, 1998.

23 Ossip-Klein DJ, Giovino GA, Megahed N, et al. Effects of a smokers' hotline: results of a 10-county self-help trial. $\mathcal{F}$ Consult Clin Psychol 1991;59:325-32.
24 Stevens C, Zhu S-H. Listen first, advertise second: lessons learned from an underserved population. Paper presented at Orlando, Florida, 1999 .

25 Fiore MC, Bailey WC, Cohen SJ, et al. Smoking cessation. Clinical Practice Guideline No 18. Rockville, Maryland: US Department of Health and Human Services, Public Health Service, Agency for Health Care Policy and
Research, April 1996. (AHCPR Publication No 960692.); <http://text.nlm.nih.gov>

26 Thorndike AN, Rigotti NA, Stafford RS, Singer DE. National patterns in the treatment of smokers by physicians. FAMA 1998;279:604-8.

27 Hollis J. Physician based cessation. In Burns D, ed. Population-based approach to smoking cessation. US Department of Health and Human Services, NCI Monograph. In press.

28 Lichtenstein E, Hollis J. Patient referral to a smoking cessation program: who follows through? $\mathcal{f}$ Fam Pract 1992;34:739-44.

29 Zhu S-H, Tedeschi G, Anderson CM, et al. Telephone counseling as adjuvant treatment for nicotine replacement therapy in a "real-world" setting. Prev Med In press.

30 Kelch D. Tobacco cessation in managed care settings: a call to action. Prepared for the next generation: California Tobacco Control

31 Nelson C, Woodwell D. National ambulatory medical care survey: 1993 summary. Series 13: Data from the National Health Survey. Vital Health Stat 1998;136:1-99.

32 US Department of Health and Human Services. The surgeon general's 1989 report on reducing the health consequences of smoking: 25 years of progress. MMWR Morbid Mortal Wkly Rep 1989;38:1-32.

33 Zhu S-H, Rosbrook B, Anderson CM, et al. The demographics of help-seeking for smoking cessation in California and the role of the California Smokers' Helpline. Tobacco Control 1995;4:S9-15.

34 Fagerstrom KO, Schneider N. Measuring nicotine dependence: a review of the Fagerstrom tolerance questionnaire. $\mathcal{F}$ Behav Med 1989;12:159-82.

35 Prochaska JO, DiClemente CC, Velicer WF, et al. Standardized, individualized, interactive, and personalized self-help
programs for smoking cessation. Health Psychol 1993; programs for

36 Zhu S-H, Anderson CM, Tedeschi G, et al. The California Smokers' Helpline: five years of experience. A Report to the California State Health Department, Tobacco Control Section Sacramento, CA: California Department of Health and Human Services, 1998.

37 Viswesvaran S, Schmidt FL. A meta-analytic comparison of the effectiveness of smoking cessation methods. F $A p p l$ Psych 1992;77:554-61.

38 Zhu S-H, Melcer T, Sun J, et al. Smoking cessation with and without assistance: a population-based analysis. Am f Prev Med In press.

39 Curry SJ. Self-help materials. In Burns D, ed. Populationbased approach to smoking cessation. US Department of Health and Human Services. NCI Monograph. In press. 